\title{
Sequence Mixup for Zero-Shot Cross-Lingual Part-Of-Speech Tagging
}

\author{
Megh Thakkar \\ BITS, Pilani \\ megh. 1211 @gmail.com \\ Ramit Sawhney \\ ShareChat \\ ramitsawhneyesharechat. co
}

\author{
Vishwa Shah \\ BITS Pilani, K. K. Birla Goa Campus \\ f20180109@goa.bits-pilani.ac.in
}

\begin{abstract}
There have been efforts in cross-lingual transfer learning for various tasks. We present an approach utilizing an interpolative data augmentation method, Mixup, to improve the generalizability of models for part-of-speech tagging trained on a source language, improving its performance on unseen target languages. Through experiments on ten languages with diverse structures and language roots, we put forward its applicability for downstream zeroshot cross-lingual tasks.
\end{abstract}

\section{Introduction}

Recently, neural network models have obtained state-of-the-art results in part-of-speech (POS) tagging tasks across multiple languages. Since $n$ merous languages lack suitable corpora annotated with POS labels, there have been efforts to-design models for cross-lingual transfer learr ang. lingual learning enables us to utilizeru nn corpora of a source language to iam mo "s that are effective over a different a languas interpolative data augmentatio me ds have been proposed to mitigate $\mathrm{c}$ erntting in $\mathrm{n}$. $1 \mathrm{~s}$ in the absence of enough ing ata. Sequence-based mixup (Chen et al., 20_ is an it erpolative data augmentation $\mathrm{d}$ for $\mathrm{m}$, , entity recognition. However, e $\mathrm{m}$ hads he not been explored for cross-lingu ansiurability.

Interpolative a augmentation methods are aimed at increasing the diversity of the training distribution and, as a result, improving the generalizability of underlying models. We leverage this capability of sequence Mixup (Seq. Mixup) to capture rich linguistic information for cross-lingual transferability of POS tagging tasks for ten languages with different structures and language roots. To this end, we first measure the dataset level cosine similarity across languages, defined as the average of sentence-level embedding of dataset samples. This gives an overview of the syntactical

\author{
Debdoot Mukherjee \\ ShareChat \\ debdoot.iitd@gmail.com
}

and semantical relationship among the different languages. We then finetune multilingual models over a source language using sequence Mixup and evaluate it across a target language for varying language similarities, pro 119 ence-based interpolative data augmen on. We à evaluate sequence Mixup on co nbina ns of milar and dissimilar language and verify tr nsferability on various target

\section{Metho logy}

Mixup ang c. 2018) is a data augmentation tect rque that gen res virtual training samples fro convex ambinations of individual inputs and lab For a $p$, r of data points $(x, y)$ and $\left(x^{\prime}, y^{\prime}\right)$, Mixup_ s a new sample $(\widetilde{x}, \widetilde{y})$ by interpolating ata points using a ratio $\lambda$, sampled from a Beta stribution, where $\widetilde{x}=\lambda \cdot x+(1-\lambda) \cdot x^{\prime}$ and corresponding mixed label $\widetilde{y}=\lambda \cdot y+(1-\lambda) \cdot y^{\prime}$. We perform Mixup over the latent space representations for interpolating sequences. Pair of sentences $(x, y)$ and $\left(x^{\prime}, y^{\prime}\right)$ are randomly sampled and interpolated in the hidden space using a $L$-layer encoder $f(:, \theta)$. The hidden layer representations for $x$ and $x^{\prime}$ upto the $k^{\text {th }}$ layer are given as,

$$
\begin{array}{ll}
h_{l}=f_{l}\left(h_{l-1} ; \theta\right), & l \in[1, k] \\
h_{l}^{\prime}=f_{l}\left(h_{l-1}^{\prime} ; \theta\right), & l \in[1, k]
\end{array}
$$

At the $k^{t h}$ layer, the hidden representations of each token in $x$ are linearly interpolated with each token in $x^{\prime}$. After this, $\widetilde{h}_{k}$ is fed to the upper layers,

$$
\begin{array}{r}
\widetilde{h}_{k}=\lambda h_{k}+(1-\lambda) h_{k}^{\prime} \\
\widetilde{h}_{l}=f_{l}\left(\widetilde{h}_{l-1} ; \theta\right), \quad l \in[k+1, L]
\end{array}
$$

We evaluate the performance of zero-shot learning on sequence Mixup, where the model is trained on one source language or a set of source languages and evaluated on a target language. To choose the source and target, for each language we average sentence level embeddings of dataset instances and use the average embeddings as language representation. Using these representations, we find the cosine similarity among the languages as shown in 
Figure 1. This approach can be extended for tasks on under-resourced languages, where models can be trained on a similar high-resourced language or a set of languages.

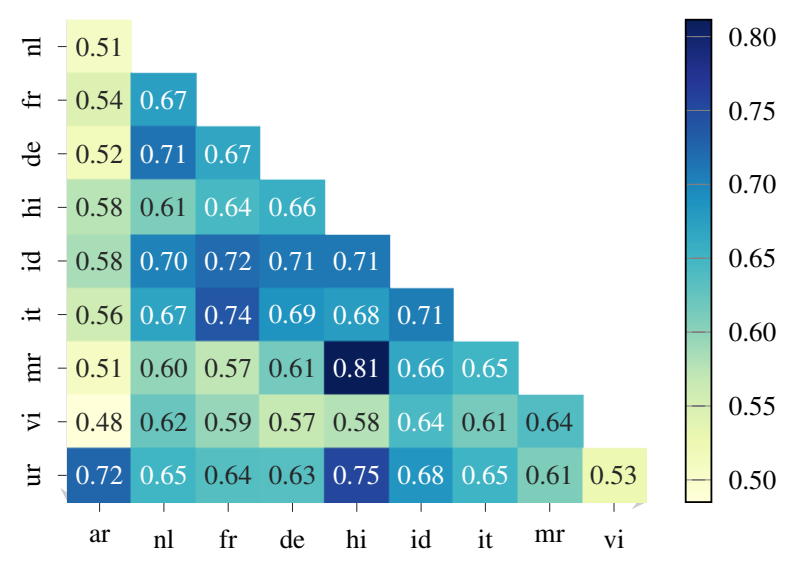

Figure 1: Cosine Similarity of the ten languages.

\section{Experiments}

We evaluate our approach on POS tagging with datasets from the Universal Dependencies (UD) dataset ${ }^{1}$ for ten different languages - Arabic (ar), Dutch (nl), French (fr), German (de), Hindi (hi), Indonesian (id), Italian (it), Marathi (mr), Vietnamese (vi) and Urdu (ur). For each experiment, we us 800 sentences for each source language for trair ing, 100 sentences for validation and test each from the target language for evaluation. $\mathrm{B}^{\mathrm{T}}$ asemultilingual-cased (mBERT) has be used scan encoder in sequence Mixup and $f(\mathrm{ob}$, $1 \mathrm{~g}$ th. embeddings to evaluate cosine milarity.

Training Setup: The lear ng is $5 \mathrm{e}-5$ with Adam optimizer and ba size 16. 1/ hyperparameters are selected ased on validati in $\mathrm{F} 1$-score.

\subsection{Single Lanouage nsfer}

We train the rodel $n$ a sing source language and a different ta dataset to evaluate its performance, as own in Table 1. As sequence Mixup trains on il erpolated sequences, it regularizes the model and prevents overfitting, outperforming mBERT. For the target language Italian, we observe higher F1 when the source language is French and lower scores for source languages Hindi and Arabic. This is in line with the trend observed in Fig 1, where the cosine similarity for the language pair (French, Italian) is highest, lower for (Hindi, Italian) and lowest for (Arabic, Italian). We observe large improvements when sequence

\footnotetext{
${ }^{1}$ https://universaldependencies.org/
}

Mixup is applied over dissimilar languages, validating that Mixup is able to generate more diverse input samples which intersect with the target language structure and semantics.

\begin{tabular}{llrr}
\hline Source & \multicolumn{1}{c}{ Target } & mBERT & Seq. Mixup \\
\hline \multicolumn{4}{c}{ High Similarity } \\
French & Italian & 94.52 & 94.75 \\
Indonesian & Vietnamese & 56.08 & 56.34 \\
German & Dutch & 85.32 & 85.48 \\
Hindi & Marathi & 64.41 & 64.97 \\
Urdu & Arabic & 44.61 & 47.38 \\
\hline \multicolumn{4}{c}{ Low Similarity } \\
Hindi & Italian & 58.63 & 63.71 \\
French & Arabic & 39.63 & 40.55 \\
Arabic & Italian & 25.41 & 28.42 \\
\hline
\end{tabular}

Table 1: F1-scores for POS tag ng on Mixup and mBERT (mean of 10 runs) rovemen are shown with blue $(\uparrow)$ over $\mathrm{mBF}^{\mathrm{N}} \mathrm{T}$.

\subsection{Multi Langy re $T$, nsfer}

To extend our a srim we coose a pair of languages on the mo trained and present the resuk in e 2. This helps to infer in what man -1 ditiona $n$ zuage data impacts the perfor lance. Languages Dutch, German and French ha high cos e similarity, leading to larger improv nt fo the Dutch language compared to sinlanguage transfer. For target language Italian, ( 1 decreases when trained on both Arabic and French data; this can be reasoned by the low ssine similarity of Arabic and Italian language.

\begin{tabular}{llrr}
\hline Source & Target & Single Source & Dual Source \\
\hline German+French & Italian & 94.75 & 94.85 \\
French+German & Dutch & 85.48 & 86.11 \\
Arabic+French & Italian & 94.75 & 28.00 \\
Arabic+Hindi & Marathi & 64.97 & 30.07 \\
\hline
\end{tabular}

Table 2: F1-scores for POS tagging on Seq. Mixup and mBERT (mean of 10 runs) when trained on two source languages (New+Original). Improvements are shown with blue $(\uparrow)$ and poorer performance with red $(\downarrow)$.

\section{Conclusion}

We analyze interpolative regularization based data augmentation over tokens for zero-shot crosslingual transfer of part-of-speech tagging across ten languages. Through extensive experiments over languages with varying syntactic and semantic structures on single and pair of languages, we pave the way for using interpolative data augmentation to improve the generalizability of neural networks for zero-shot transfer learning on downstream tasks. 


\section{References}

Jiaao Chen, Zhenghui Wang, Ran Tian, Zichao Yang, and Diyi Yang. 2020. Local additivity based data augmentation for semi-supervised NER. In Proceedings of the 2020 Conference on Empirical Methods in Natural Language Processing (EMNLP), pages 1241-1251, Online. Association for Computational Linguistics.

Hongyi Zhang, Moustapha Cisse, Yann N. Dauphin, and David Lopez-Paz. 2018. mixup: Beyond empirical risk minimization. In International Conference on Learning Representations.

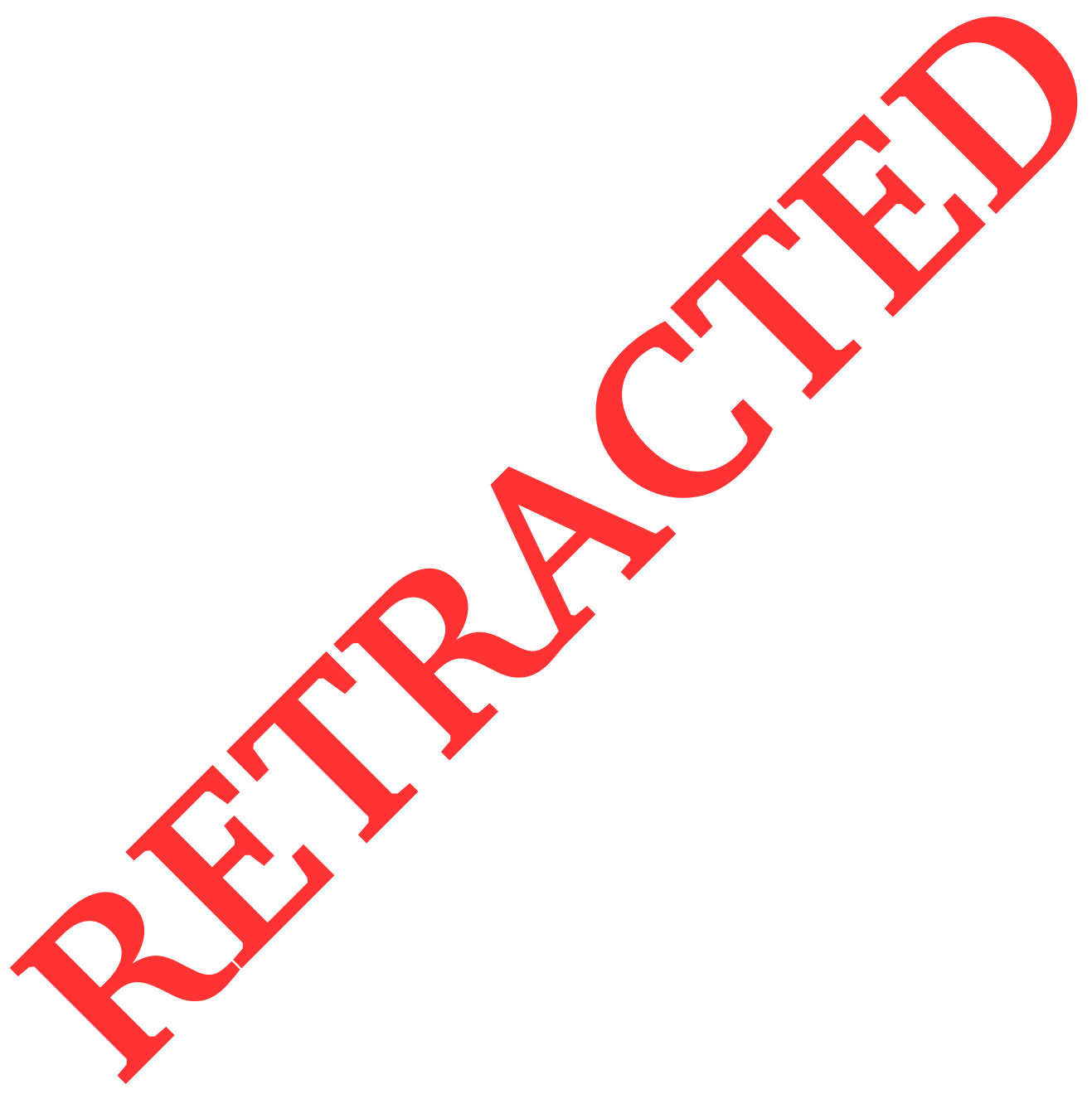

